\title{
On the Architecture and Performance of an FFT-Based Spread-Spectrum Downlink RAKE Receiver
}

\author{
Shin-Yuan Wang and Chia-Chi Huang
}

\begin{abstract}
This paper describes a spread-spectrum downlink RAKE receiver that computes a data detection in the frequency domain. We assume a pilot signal is transmitted with data signals for channel sounding. The pilot signal does not degrade the receiver bit error rate (BER) performance because the receiver estimates the pilot signal and subtracts the estimated pilot signal from the received signal before data detection. A spreading code matched filter, a channel matched filter, and a sounding receiver are implemented by fast Fourier transform (FFT)-based matched filtering and integrated in a unified architecture. Monte Carlo simulation is used to evaluate the receiver BER performance in both a static channel and a mobile radio channel. Simulation results show that the RAKE receiver performs well in both kinds of channels.
\end{abstract}

Index Terms-Fast Fourier transform (FFT)-based matched filtering, RAKE receivers, spread spectrum.

\section{INTRODUCTION}

W IRELESS cellular communications around the world are moving toward code-division multiple-access (CDMA) systems in almost all third-generation approaches because a CDMA system achieves much higher bandwidth efficiency on a limited radio spectrum [1]-[3]. It has been claimed that a CDMA system ideally provides a much larger system capacity gain over other access methods, such as frequency-division multiple access and time-division multiple access methods [4]-[5]. In general, a CDMA system uses a RAKE receiver to combine the received signal energy from different radio propagation paths in order to combat the effect of multipath fading [6]. This multipath diversity provides a robust communication channel because when some paths fade, communication is still possible through other nonfading paths.

Numerous types of RAKE receivers have been suggested in the literature [6]-[11]. Coherent detection with maximal ratio combining is a common strategy for implementing RAKE receivers. In more detail, coherent RAKE receivers can be implemented by either a matched filter-based RAKE receiver requires a spreading code matched filter for code despreading and a transversal filter for matching to channel impulse response. The spreading code matched filter can be implemented either at intermediate frequency band using a surface acoustic wave (SAW) filter or at baseband using a digital matched filter. After

Manuscript received June 26, 1999; revised October 15, 1999.

The authors are with the Department of Communication Engineering, National Chiao Tung University, Hsinchu, Taiwan, R.O.C.

Publisher Item Identifier S 0018-9545(01)01924-7. code despreading, a digital transversal filter at baseband can be used to combine the received signal energy from different paths. The main drawback of this SAW filter approach comes from the fact that a SAW device cannot be easily integrated with a baseband digital transversal filter in an integrated circuit (IC). Therefore, the digital spreading code matched filter approach is a preferable choice when the RAKE receiver is to be implemented in an IC. Although current IC technology provides a large computation capability, it is still hard to implement a digital matched filter-based RAKE receiver on a single chip, especially when a long spreading code is used. An alternative method is to implement a RAKE receiver using a bank of correlators. Each correlator is used to detect a received signal path separately. The number of the correlators in the correlator bank is typically three or four. Although the correlator bank approach has low implementation complexity, it needs extra searchers to search for different multipath signals and a tracking loop for each correlator.

Within a RAKE receiver, the instantaneous channel impulse response has to be estimated for channel matching purpose. There are two methods to estimate a channel impulse response. One method uses the spectrum spread data signal to estimate the channel impulse response through a decision-directed approach [8]. This approach does not require transmitting an extra pilot signal for channel sounding but suffers from the error propagation problem. The other method sounds a channel by transmitting a pilot symbol or a pilot signal with data [9], [10]. The extra pilot symbol or pilot signal usually degrades the system performance due to the extra power used. In general, with both methods, a channel sounding receiver is needed to estimate the channel impulse response. In a CDMA system with a pilot signal, a downlink receiver needs to monitor the pilot signal continuously. The power of the pilot signal should be large enough for base-station monitoring in surrounding cells. The interference from all the pilot signals will degrade the system performance. However, a well-designed pilot interference cancellation technique can be used to reduce this effect [12].

In this paper, we propose a RAKE receiver architecture that computes a data detection in the frequency domain using fast Fourier transform (FFT)-based matched filters. Here, we consider a downlink CDMA system implemented with equal-length short codes, including a pilot spreading code and many data spreading codes. For this system, we integrate a spreading code matched filter, a channel matched filter, and a sounding receiver within a single receiver architecture. In order 
to reduce the needed computation power, the three matched filters share the same FFT computation result from the received signal. The RAKE receiver also adopts a pilot interference cancellation technique to reduce its interference effect. Another feature of our RAKE receiver is that the task of multipath searching and tracking is done on a symbol-by-symbol basis in the sounding receiver instead of using sliding correlators and tracking loops.

This paper describes the operation and evaluates the performance of the FFT-based RAKE receiver architecture. We consider only the case of one user and treat the interference from many other users as Gaussian noise in our computer simulation [13]. In Section II, we describe the transmitted spread-spectrum signal. In Section III, we present the channel models used in our simulation. The details of our RAKE receiver architecture whose function includes channel sounding, pilot interference cancellation, and data signal detection are covered in Section IV. Computer simulation results are presented in Section V. A final conclusion is given in Section VI.

\section{The TransmitTED SPREAD-SPECTRUM SignAL}

Our transmitter is a direct-sequence spread-spectrum (DS-SS) modulator. The transmitted DS-SS signal consists of a data signal and a pilot signal. The data signal is modulated by binary phase-shift keying (BPSK) and multiplied with a data signal spreading code. The pilot signal is unmodulated and multiplied with a pilot signal spreading code. Here, both the data signal spreading code and the pilot signal spreading code are assumed to be equal-length short codes and synchronized with information bits, that is, the periods of both spreading codes overlap with the information bit period. The data signal and the pilot signal are combined to become a transmitted signal $s(t)$, which can be represented by

$$
s(t)=\Re\left\{\tilde{s}(t) \exp \left(j \omega_{c} t+\theta\right)\right\}
$$

where

$\Re\{\cdot\}$ real part notation;

$\tilde{s}(t) \quad$ equivalent baseband signal of $s(t)$;

$\omega_{c} \quad$ carrier frequency;

$\theta \quad$ carrier phase.

The $i$ th symbol of $\tilde{s}(t)$ is

$$
\begin{aligned}
\tilde{s}^{(i)}(t)= & \sqrt{2 P_{p}} \sum_{n=1}^{N} c_{p, n} U\left(t-n T_{c}\right)+\sqrt{2 P_{d}} d^{(i)} \sum_{n=1}^{N} \\
& \cdot c_{d, n} U\left(t-n T_{c}\right)
\end{aligned}
$$

where

$P_{p} \quad$ transmission power of the pilot signal;

$c_{p, n} n$th chip of the pilot signal spreading code which has $N$ chips per period;

$P_{d} \quad$ transmission power of the data signal;

$d^{(i)} \quad$ ith information bit, $d^{(i)} \in\{-1,1\}$;

$c_{d, n} n$th chip of the data signal spreading code, which has $N$ chips per period;

$T_{C} \quad$ chip period of the spreading codes;

$U(t)$ rectangular pulse function defined by $U(t)=1$ for $0 \leq t \leq T_{c}$, and $U(t)=0$ elsewhere.

\section{Channel Model}

The performance of the RAKE receiver was evaluated in two static channels and a mobile radio channel. The two static channels include an additive white Gaussian noise (AWGN) channel and a fixed two-path channel. The multiple-access interference (MAI) from other users and the thermal noise are lumped together and modeled as an equivalent AWGN. The equivalent baseband impulse response of the fixed two-path channel is assumed to be

$$
h(t)=\delta(t)+\delta(t-\tau)
$$

where $\tau$ is the excess delay of the second path. For the mobile radio channel, we consider a two-path fading channel. Its equivalent baseband impulse response is assumed to be

$$
h(t)=a_{1}(t) \delta(t)+a_{2}(t) \delta(t-\tau)
$$

where $a_{1}(t)$ and $a_{2}(t)$ are two complex gains of the two fading paths and are generated independently using Jake's fading channel model [14], which can be further expressed as the sum of $N_{f}$ sinusoidal waves

$$
a_{k}(t)=\frac{1}{\sqrt{N_{f}}} \sum_{n=1}^{N_{f}} \exp \left(j 2 \pi f_{n} t+\phi_{k, n}\right)
$$

for $k=1,2$, where

$$
f_{n}=f_{d} \cos \left(\frac{2 \pi n}{N_{f}}\right)
$$

$f_{d}$ is the maximum Doppler frequency and $\phi_{k, n}$ is the initial random phase of each wave for the $k$ th path.

The signal observed at a receiver will be

$$
r(t)=\Re\left\{\tilde{r}_{b}(t) \exp \left(j \omega_{c} t+\theta\right)\right\}+n(t)
$$

where $\tilde{r}_{b}(t)$ is the received equivalent baseband signal and $n(t)$ is the AWGN. $\tilde{r}_{b}(t)$ can be expressed as

$$
\widetilde{r}_{b}(t)=h(t) \otimes \tilde{s}(t)
$$

where the symbol $\otimes$ denotes convolution. From the convolution theorem of Fourier transform, we can write (8) equivalently in the frequency domain as

$$
\tilde{R}_{b}(f)=H(f) \tilde{S}(f)
$$

where $\tilde{R}_{b}(f), H(f)$, and $\tilde{S}(f)$ are Fourier transforms of $\tilde{r}_{b}(t)$, $h(t)$, and $\tilde{s}(t)$, respectively.

\section{The FFT-BASEd SPREAD-SPECTRUM DOWNLINK RAKE RECEIVER}

A simplified block diagram of an FFT-based spread-spectrum downlink RAKE receiver is shown in Fig. 1. A radio-frequency (RF) signal is received from an antenna and converted to an equivalent baseband signal by the RF front end and the $I-Q$ demodulator. We assume that the system is bandlimited to the chip rate $1 / T_{c}$. To reduce the computation load of FFT, the sampling rate of the analog-to-digital (A/D) converter is chosen to be the 


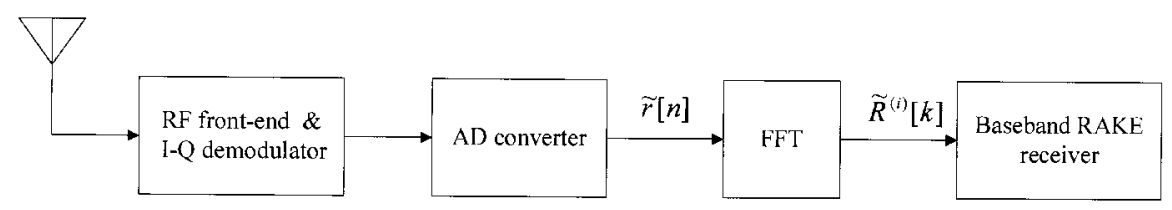

Fig. 1. Simplified block diagram of an FFT-based spread-spectrum downlink RAKE receiver.

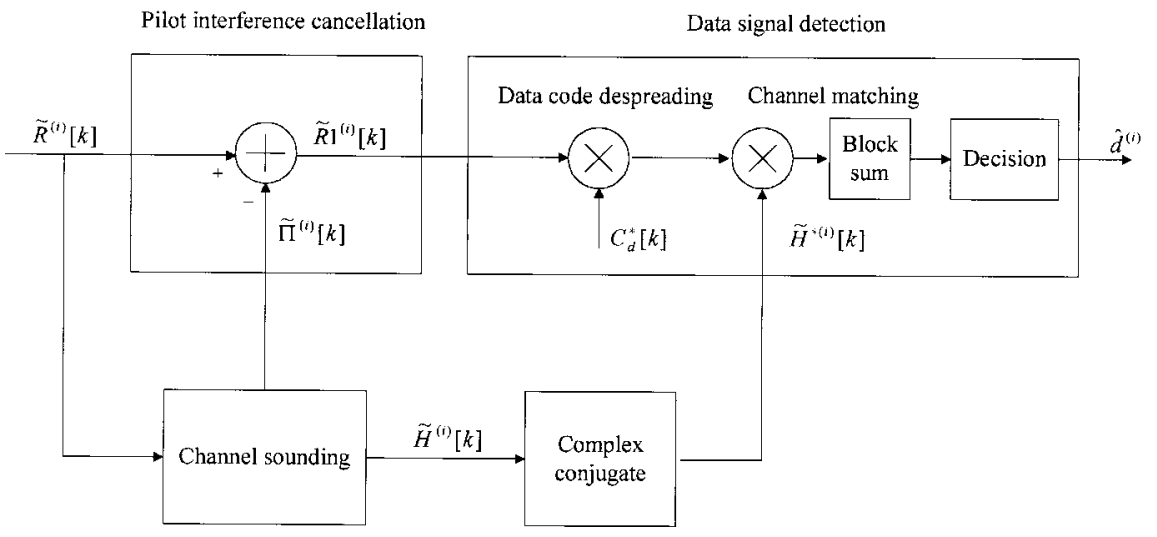

Fig. 2. The block diagram of the baseband RAKE receiver.

same as the Nyquist rate, i.e., the chip rate. As the sampling rate is the chip rate, we might not be able to sample exactly at the peak of each path signal. However, the energy of a path signal whose delay does not exactly align with the sampling time will be dispersed into adjacent samples. As a result, most of the multipath signal energy will still be preserved during the A/D conversion process [15]. After A/D conversion, the discrete time equivalent baseband signal $\tilde{r}[n]$ is segmented for $N$-point FFT computation. After segmentation, $\tilde{r}[n]$ can be expressed as

$$
\tilde{r}[n]=\sum_{i=0}^{\infty} \tilde{r}^{(i)}[n-i N]
$$

where $\tilde{r}^{(i)}[n]$ is the $i$ th segment of $\tilde{r}[n], 0 \leq n \leq N-1$. Here, we assume that the window for FFT computation is aligned with the received symbol timing. The FFT of $\tilde{r}^{(i)}[n]$ is

$$
\begin{aligned}
\tilde{R}^{(i)}[k]= & \mathcal{F F \mathcal { T }}\left\{\tilde{r}^{(i)}[n]\right\} \\
= & \sqrt{2 P_{p}} C_{p}[k] H^{(i)}[k]+\sqrt{2 P_{d}} d^{(i)} C_{d}[k] H^{(i)}[k] \\
& +N[k]
\end{aligned}
$$

for $k=0,1, \ldots, N-1$, where $\mathcal{F F} \mathcal{F}\{\cdot\}$ denotes the FFT operation and

$C_{p}[k] \quad$ FFT of the pilot signal spreading code;

$C_{d}[k] \quad$ FFT of the data signal spreading code;

$H^{(i)}[k]$ FFT of the channel impulse response for the $i$ th segmentation symbol;

$N[k] \quad$ FFT of AWGN (including interference).

After FFT computation, all signal processing is performed in the frequency domain. Fig. 2 shows the block diagram of the baseband RAKE receiver. $\tilde{R}^{(i)}[k]$ is sent to the upper arm for pilot interference cancellation and data signal detection whose function further includes data code despreading, channel matching, and data decision. $\tilde{R}^{(i)}[k]$ is also sent to the lower arm for channel sounding. Here, two methods can be used, one without path selection and the other one with path selection. The method without path selection is suggested to be used when the channel fading rate is low. The other method is more general and can be used in almost all situations.

\section{A. Channel Sounding Without Path Selection}

A mobile radio channel exhibits a wide range of channel variation rates. A slower fading channel allows a longer period to estimate the pilot signal. On the other hand, a faster fading channel allows only a shorter period for pilot signal estimation. Therefore, the time constant for pilot signal estimation theoretically should be adapted to the channel variation rate such that a channel can be tracked more closely. In any case, the time constant of the channel estimator should be much less than the coherence time of the fading channel [16].

We first consider a slow varying radio propagation channel, such as that for a wireless local loop (WLL). This kind of channel is more like a static channel, i.e., the channel does not change over a long period of time [17], [18]. In such a case, a relatively longer period can be used for pilot signal estimation since the unmodulated pilot signal can be "coherently" added during the averaging period. On the other hand, both the multiple access interference and the background noise will be averaged out during this period. Fig. 3(a) shows the block diagram for channel sounding without path selection. Here, the received signal $\tilde{R}^{(i)}[k]$ is directly used for pilot signal estimation. The estimated pilot signal $\tilde{\Pi}^{(i)}[k]$ is used for pilot interference cancellation. To avoid the data signal interference problem at the pilot interference cancellation stage, we reconstruct the data signal component using a decision feedback approach and then subtract the reconstructed data from the received signal before pilot signal estimation.

The data signal reconstruction process is shown in Fig. 3(b). The data decision output of the previously received symbol 


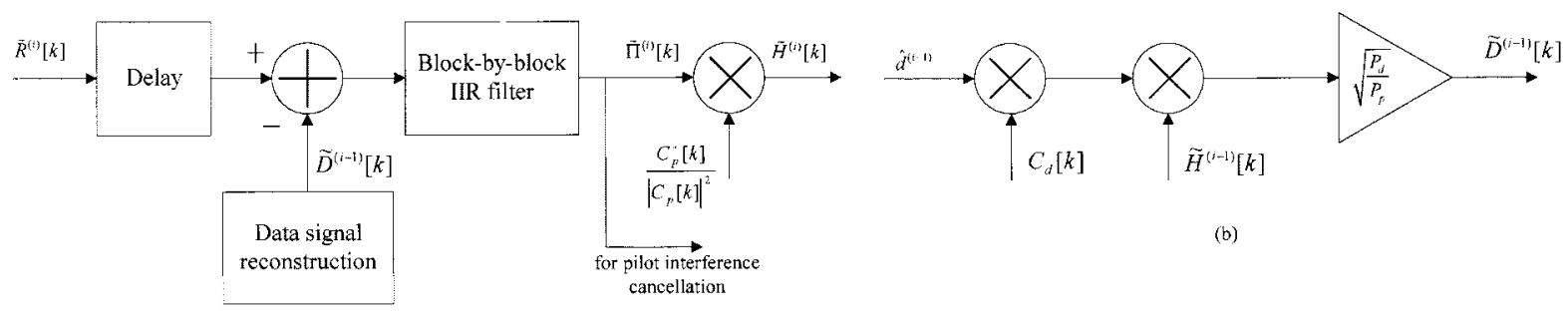

(a)

Fig. 3. (a) Channel sounding without path selection. (b) Data signal reconstruction.

$\hat{d}^{(i-1)}$ is multiplied by the FFT of the data signal spreading code $C_{d}[k]$, by the estimated channel frequency response for the previously received symbol $\tilde{H}^{(i-1)}[k]$, and then normalized by $\sqrt{P_{d} / P_{p}}$ to adjust for transmission power difference between the pilot signal and the data signal. As a result, the reconstructed data signal component of the previously received symbol becomes

$$
\tilde{D}^{(i-1)}[k]=\hat{d}^{(i-1)} C_{d}[k] \tilde{H}^{(i-1)}[k] \sqrt{\frac{P_{d}}{P_{p}}}
$$

for $k=0,1, \ldots, N-1$.

The received signal $\tilde{R}^{(i)}[k]$ is delayed for one symbol period. The delay is used to prevent the "noise matching" problem at the later channel matching stage since the estimated pilot signal is also used to estimate the channel frequency response. The reconstructed data signal $\tilde{D}^{(i-1)}[k]$ is then subtracted from the delayed version of the received signal $\tilde{R}^{(i-1)}[k]$. After that, a block-by-block first-order infinite impulse response (IIR) filter is used to estimate the pilot signal, i.e.,

$$
\tilde{\Pi}^{(i)}[k]=\alpha \tilde{\Pi}^{(i-1)}[k]+(1-\alpha)\left(\tilde{R}^{(i-1)}[k]-\tilde{D}^{(i-1)}[k]\right)
$$

for $k=0,1, \ldots, N-1$, where $\alpha$ is the time constant of the IIR filter. Substituting (11) and (12) into (13) yields (temporarily ignoring the noise term)

$$
\begin{aligned}
\tilde{\Pi}^{(i)}[k]= & \alpha \tilde{\Pi}^{(i-1)}[k]+(1-\alpha) \sqrt{2 P_{p}} C_{p}[k] H^{(i-1)}[k] \\
& +(1-\alpha) \sqrt{2 P_{d}} C_{d}[k] \\
& \cdot\left\{d^{(i-1)} H^{(i-1)}[k]-\hat{d}^{(i-1)} \tilde{H}^{(i-1)}[k] / \sqrt{2 P_{p}}\right\}
\end{aligned}
$$

for $k=0,1, \ldots, N-1$. When $\tilde{H}^{(i-1)}[k] / \sqrt{2 P_{p}}$ approaches $H^{(i-1)}[k]$ and data are detected correctly [i.e., $\left.\hat{d}^{(i-1)}=d^{(i-1)}\right]$, the IIR filter output becomes

$$
\tilde{\Pi}^{(i)}[k]=\alpha \tilde{\Pi}^{(i-1)}[k]+(1-\alpha) \sqrt{2 P_{p}} C_{p}[k] H^{(i-1)}[k]
$$

for $k=0,1, \ldots, N-1$. Let

$$
\tilde{\Pi}^{(i)}[k] \equiv C_{p}[k] \tilde{H}^{(i)}[k] .
$$

Equation (15) can be reduced to

$$
\tilde{H}^{(i)}[k]=\alpha \tilde{H}^{(i-1)}[k]+(1-\alpha) \sqrt{2 P_{p}} H^{(i-1)}[k]
$$

for $k=0,1, \ldots, N-1$. Thus, $\tilde{H}^{(i)}[k]$ is just the estimated channel frequency response and

$$
\tilde{H}^{(i)}[k]=\tilde{\Pi}^{(i)}[k] \frac{C_{p}^{*}[k]}{\left|C_{p}[k]\right|^{2}} .
$$

\section{B. Channel Sounding with Path Selection}

The channel sounding method discussed in the previous section requires relatively long average time and works well only in a slow fading channel. Otherwise, the signal-to-noise ratio (SNR) of the estimated channel frequency response will degrade and the system performance will deteriorate. In a cellular system that does not allow for long average time, we suggest the use of path selection to enhance the SNR of the estimated channel frequency response because the path selection process will utilize the processing gain advantage of a spread-spectrum system.

Fig. 4(a) shows the block diagram for channel sounding with path selection. The received signal $\tilde{R}^{(i)}[k]$ is delayed for one symbol period to avoid the "noise matching" problem. Afterwards, a simple block-by-block first-order IIR filter is used to estimate the pilot signal. The filter output is now only a coarsely estimated pilot signal

$$
\bar{\Pi}^{(i)}[k]=\alpha \bar{\Pi}^{(i-1)}[k]+(1-\alpha) \tilde{R}^{(i-1)}[k]
$$

for $k=0,1, \ldots, N-1$. Here, the data signal component is not removed from the received signal as down in Fig. 3(a) because the coarsely estimated pilot signal $\bar{\Pi}^{(i)}[k]$ is not directly used for pilot interference cancellation. The coarsely estimated pilot signal $\bar{\Pi}^{(i)}[k]$ is multiplied by $C_{p}^{*}[k] /\left|C_{p}[k]\right|^{2}$ to obtain a coarsely estimated channel frequency response $\bar{H}^{(i)}[k]$. Afterwards, we transform $\bar{H}^{(i)}[k]$ back to a coarsely estimated channel impulse response in the time domain $\bar{h}^{(i)}[n]$ using inverse FFT (IFFT), i.e.,

$$
\bar{h}^{(i)}[n]=\mathcal{I} \mathcal{F} \mathcal{F} \mathcal{T}\left\{\bar{H}^{(i)}[k]\right\}
$$

where $\mathcal{I F \mathcal { F }} \mathcal{T}\{\cdot\}$ denotes the IFFT operation.

To suppress the noise components (including interference) in $\bar{h}^{(i)}[n]$, we reserve only a few paths with large amplitudes. A threshold $A_{\mathrm{th}}$ can be defined for path selection according to the peak value of $\left|\bar{h}^{(i)}[n]\right|$. For example, $A_{\mathrm{th}}$ can be set to $10 \mathrm{~dB}$ below the peak value. To select main paths, we reserve those paths with amplitudes above $A_{\text {th }}$ and discard all the other paths 


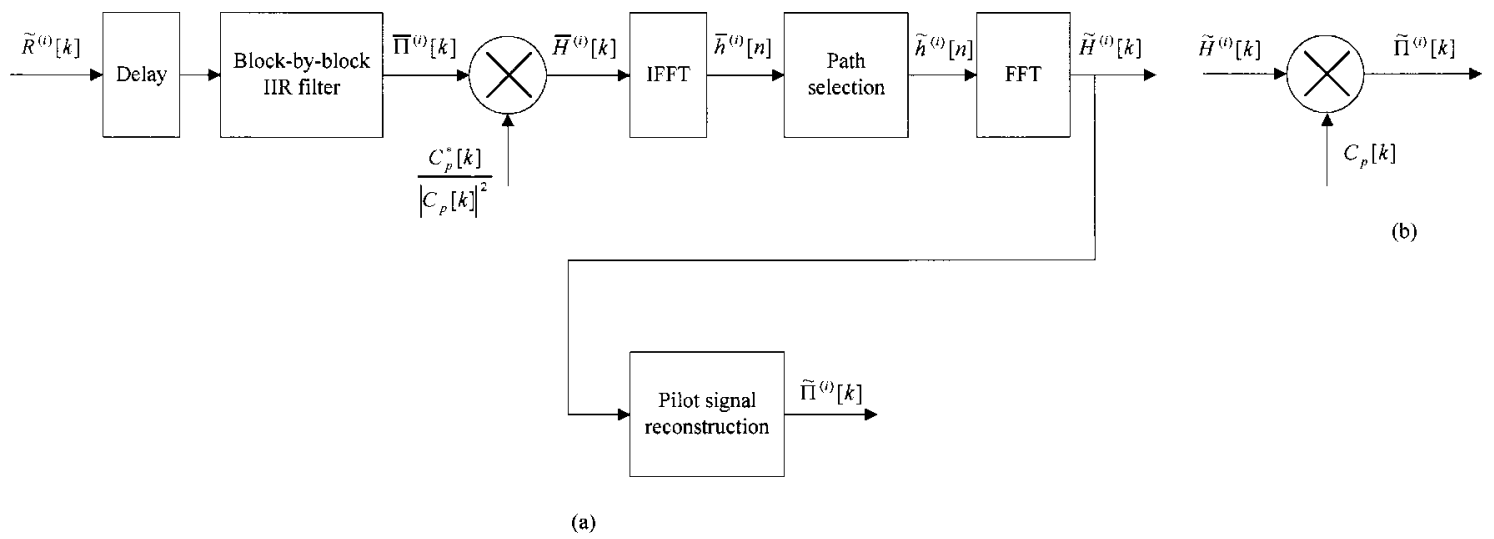

Fig. 4. (a) Channel sounding with path selection. (b) Pilot signal reconstruction.

with amplitudes below $A_{\mathrm{th}}$. As a result, an estimated channel impulse response is

$$
\tilde{h}^{(i)}[n]= \begin{cases}0, & \text { if }\left|\bar{h}^{(i)}[n]\right|<A_{\mathrm{th}}, \\ \bar{h}^{(i)}[n], & \text { if }\left|\bar{h}^{(i)}[n]\right| \geq A_{\mathrm{th}}\end{cases}
$$

for $n=0,1, \ldots, N-1$. After path selection, $\tilde{h}^{(i)}[n]$ is transformed back to an estimated channel frequency response, $\tilde{H}^{(i)}[k]$, using FFT, i.e.,

$$
\tilde{H}^{(i)}[k]=\mathcal{F F \mathcal { T }}\left\{\tilde{h}^{(i)}[n]\right\} .
$$

A conventional RAKE receiver first uses a sliding correlator to search for the multipath delay profile and a few tracking loops to acquire the channel parameters, such as the delays and the complex gains of the main paths. Then it uses multiple RAKE fingers to detect data. Unlike a conventional RAKE receiver, both path searching and tracking are done implicitly in our channel sounding subsystem because it searches and tracks all paths within one symbol period in the time domain. In a cellular system, the multipath structure may change abruptly, for example, when a vehicle turns around a street corner. The sliding correlator of a conventional RAKE receiver suffers from the estimation delay involved in searching for the multipath delay profile. Therefore, a conventional RAKE receiver may lose track of some newly arrived or disappeared paths, and this will degrade the system performance. At contrast, our RAKE receiver induces much less estimation delay since our path searching and tracking are done on a symbol-by-symbol basis and it can be quickly adapted to the change of the channel.

We can also use the estimated channel frequency response to reconstruct the estimated pilot signal. Fig. 4(b) shows the pilot signal reconstruction process. The estimated pilot signal is

$$
\tilde{\Pi}^{(i)}[k]=C_{p}[k] \tilde{H}^{(i)}[k]
$$

for $k=0,1, \ldots, N-1$, where $C_{p}[k]$ is the FFT of the pilot signal spreading code.

\section{Pilot Interference Cancellation}

To improve the system performance, our RAKE receiver adopts pilot interference cancellation. The estimated pilot signal is subtracted from the received signal before data detection (see Fig. 2). After pilot interference cancellation, the remaining signal is

$$
\tilde{R} 1^{(i)}[k]=\tilde{R}^{(i)}[k]-\tilde{\Pi}^{(i)}[k]
$$

for $k=0,1, \ldots, N-1$, where $\tilde{R} 1^{(i)}[k]$ is the remaining signal for the $i$ th received symbol and $\tilde{\Pi}^{(i)}[k]$ is the estimated pilot signal for the $i$ th received symbol.

\section{Data Signal Detection}

The data signal detection block of the baseband RAKE receiver includes data code despreading, channel matching, and data decision, as shown in Fig. 2.

After pilot interference cancellation, the remaining signal $\tilde{R} 1^{(i)}[k]$ is fed to a data code despreading matched filter in the frequency domain [19], [20]. Unlike implementing a time-domain code matched filter with a transversal filter, only a multiplier is used in implementing a frequency-domain code matched filter (the theory of FFT-based code despreading is covered in Appendix A). $\tilde{R} 1^{(i)}[k]$ is simply multiplied by the complex conjugate of the FFT of the data signal spreading code $C_{d}^{*}[k]$ for data code despreading.

In a multipath channel, intersymbol interference (ISI) will still degrade the system performance (more detailed discussion is presented in Appendix A). This ISI originates from the delayed paths of the previous symbol because each segment for FFT computation is exactly one symbol period long. This ISI effect can be neglected when the symbol period is much longer than the channel delay spread.

Finally, a channel matched filter is used to combine the signal power from different paths for data detection. The channel matched filter is in effect a maximal-ratio combiner for multipath signals, which is also implemented in the frequency domain (see Appendix B). The coefficients of the channel matched filter are obtained from the complex conjugate of the estimated channel frequency response $\tilde{H}^{*(i)}[k]$. The data code despreading output $\tilde{R} 1^{(i)}[k] C_{d}^{*}[k]$ is multiplied by $\tilde{H}^{*(i)}[k]$ to generate a frequency-domain channel matching output $\tilde{R} 1^{(i)}[k] C_{d}^{*}[k] \tilde{H}^{*(i)}[k]$. A conventional method applies an IFFT to yield a time-domain channel matching output $\Gamma_{\mathrm{ch}}^{(i)}[n]$, i.e.,

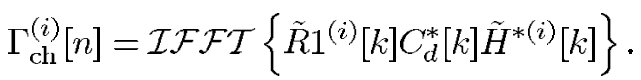


TABLE I

RECEIVER PARAMETERS

\begin{tabular}{ll}
\hline carrier frequency & $2 \mathrm{GHz}$ \\
code length, $\mathrm{N}$ & $512($ processing gain $\approx 27 \mathrm{~dB})$ \\
bit rate & $19.2 \mathrm{kbit} / \mathrm{sec}($ chip rate $\approx 10 \mathrm{MHz})$ \\
modulation & BPSK DSSS
\end{tabular}

Because the whole signal-processing chain of our RAKE receiver is done on a symbol-by-symbol basis and because the pilot signal and the data signal are synchronized, the exactly channel matched point in the channel matching output $\Gamma_{\mathrm{ch}}^{(i)}[n]$ is just the first point $\Gamma_{\mathrm{ch}}^{(i)}[0]$. According to the property of FFT, $\Gamma_{\mathrm{ch}}^{(i)}[0]$ can be calculated by averaging the frequency-domain channel matching output $\tilde{R} 1^{(i)}[k] C_{d}^{*}[k] \tilde{H}^{*(i)}[k]$ within one symbol period, i.e.,

$$
\Gamma_{\text {ch }}^{(i)}[0]=\frac{1}{N} \sum_{k=0}^{N-1} \tilde{R} 1^{(i)}[k] C_{d}^{*}[k] \tilde{H}^{*(i)}[k] .
$$

After channel matching, we can detect the data information $\hat{d}^{(i)}$ by determining whether the real part of $\Gamma_{\mathrm{ch}}^{(i)}[0]$ is greater or less than zero, as BPSK is used here as an example. More generally, other data modulation schemes can also be used with our RAKE receiver.

\section{Computer Simulation Results}

To evaluate the performance of the FFT-based RAKE receiver, a series of computer simulations were carried out under different channel conditions. Table I shows the simulation parameters of our RAKE receiver. Here, we assume that each path has the same average $\operatorname{SNR} \gamma=E_{b} / \sigma_{n}^{2}$, where $E_{b}$ is the average bit energy of the data signal for each path and $\sigma_{n}^{2}$ is the variance of the noise including MAI and thermal noise. Suppose that $L$ is the total number of paths ( $L=1$ for an AWGN channel, $L=2$ for both a fixed two-path channel and a two-path fading channel). The total average SNR is $\Upsilon_{T}=L \gamma$. We did not include the pilot signal power in $\Upsilon_{T}$ calculation because

1) in our RAKE receiver pilot interference cancellation is used;

2) the pilot signal is shared for all users;

3) we want to compare our simulation results with theoretically optimal results.

The system performances were shown by plotting the average bit error probability versus $\Upsilon_{T}$. In our simulations, the RAKE receiver was assumed to be a bandlimited system with an equivalent baseband bandwidth $1 / T_{c}$, and the sampling rate was exactly the chip rate.

Figs. 5 and 6 show the system bit error rate performances for the channel sounding method without path selection in an AWGN channel and a fixed two-path channel respectively. The effect of the pilot power ratio was examined in Fig. 5. Fig. 6 shows the effect of ISI. In Fig. 7, we compared the system performances in an AWGN channel using two different channel sounding methods (with and without path selection). Finally, both channel sounding methods were simulated in a two-path fading channel, and the results are shown in Figs. 8 and 9. In

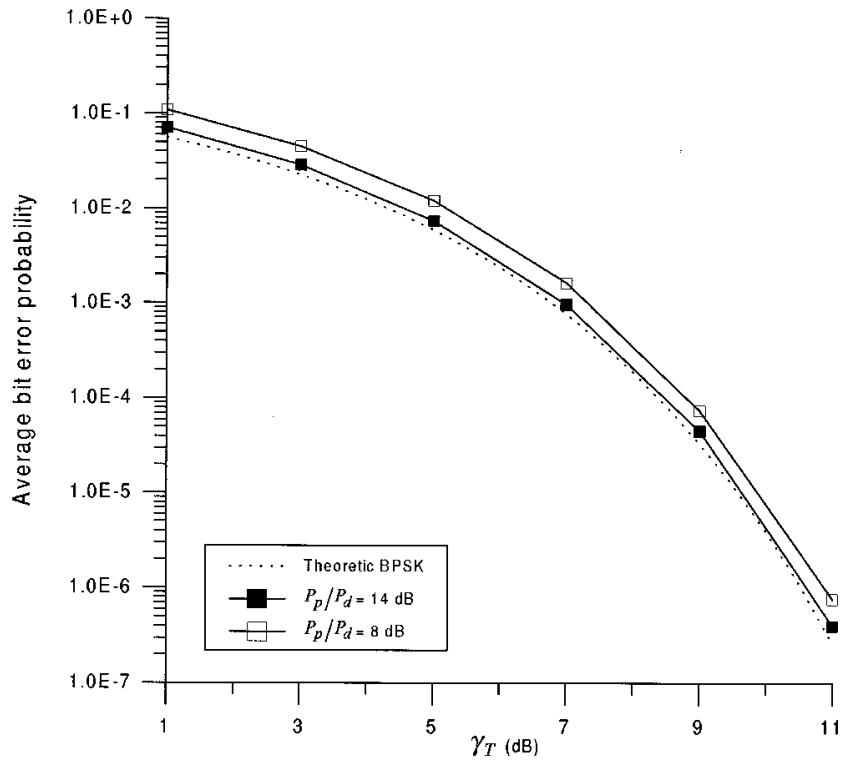

Fig. 5. Average bit error probability versus $\Upsilon_{T}$ at different pilot-to-data signal power ratio in an AWGN channel when channel sounding without path selection was used $(\alpha=0.99)$

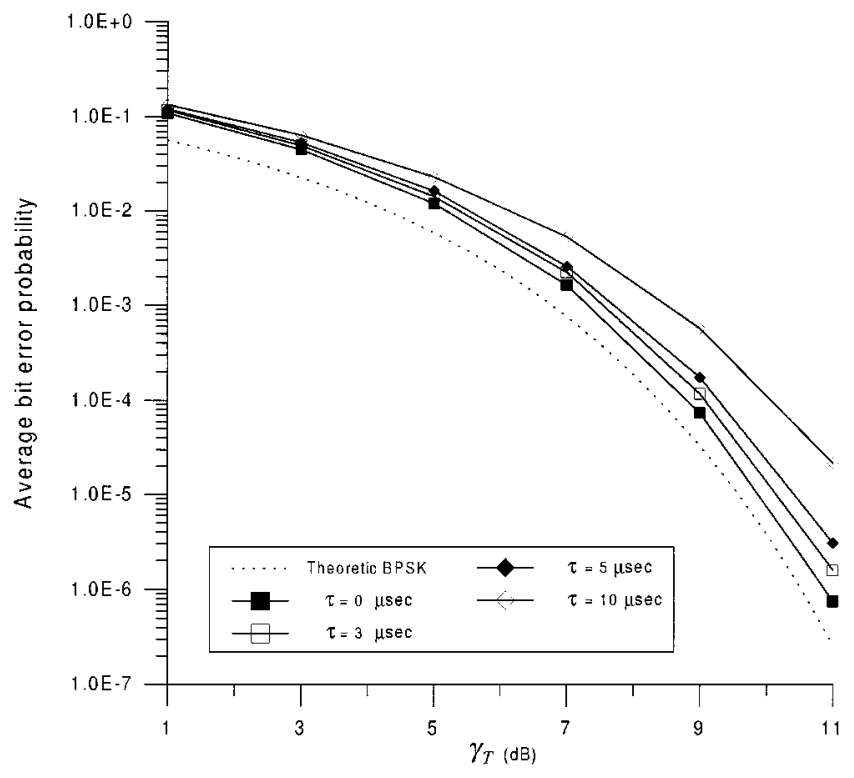

Fig. 6. Average bit error probability versus $\Upsilon_{T}$ at different excess delays of a fixed two-path channel when channel sounding without path selection was used $\left(P_{p} / P_{d}=8 \mathrm{~dB}, \alpha=0.99\right)$.

these figures, all the simulated system performances were compared with the theoretic bit error probability achievable for coherent BPSK demodulation at a RAKE receiver output [21, pp. 237-240].

Fig. 5 shows the average bit error probability versus $\Upsilon_{T}$ in an AWGN channel at different values of the pilot power ratio. We consider two cases: $P_{p} / P_{d}$ being $14 \mathrm{~dB}$ and $8 \mathrm{~dB}$. That is, suppose there are 100 channels with $100 \%$ activity and all channels are of equal power. The pilot power ratio of 14 and $8 \mathrm{~dB}$ represents $20 \%$ and $6 \%$ of the total downlink power, respectively. The time constant of the IIR filter (in Fig. 3), $\alpha$, was set to 0.99 , i.e., the average period is about 100 symbols. From Fig. 5, 


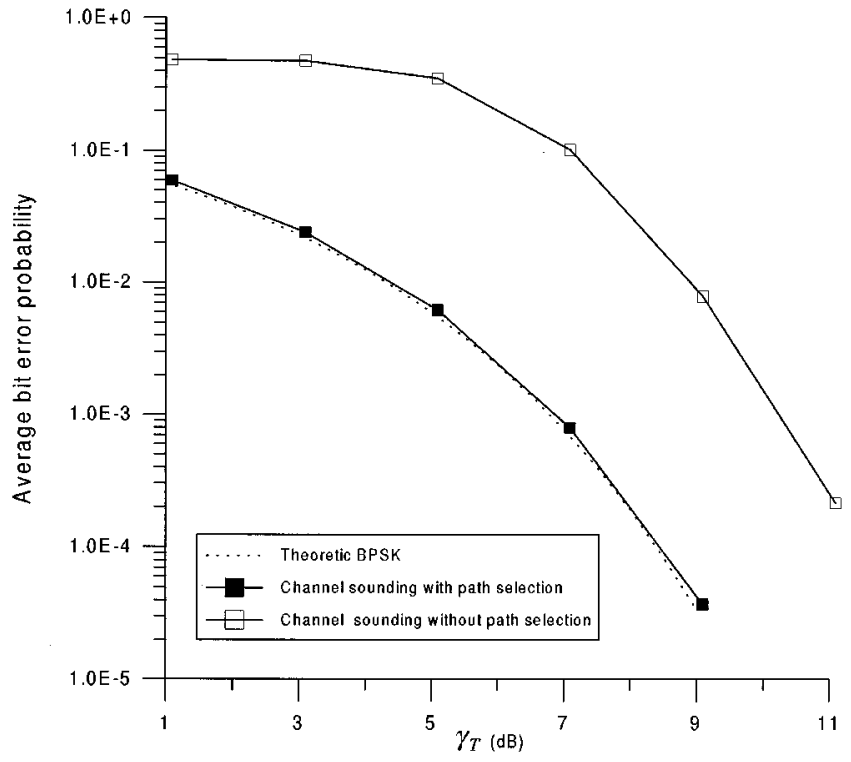

Fig. 7. Average bit error probability versus $\Upsilon_{T}$ for channel sounding with and without path selection in an AWGN channel $\left(P_{p} / P_{d}=8 \mathrm{~dB}, \alpha=0.9\right)$.

it can be observed that both cases introduce little performance degradation since the large time constant of the IIR filter provides enough SNR for the channel estimation. We also note that the pilot signal itself does not degrade the system performance because the pilot interference is almost totally cancelled out in our RAKE receiver. Note that at average bit error probability of $10^{-3}$ and when $P_{p} / P_{d}$ is $8 \mathrm{~dB}$, the power penalty (as compared with the theoretic BPSK case) is only about $0.5 \mathrm{~dB}$.

Different from the conventional RAKE receiver, the ISI effect appears in an FFT-based RAKE receiver due to the segmentation process for FFT computation. The ISI effect was examined in Fig. 6. Here, we simulated the cases in which the excess delay of the second path was 30,50, and 100 chips, i.e., the delay $\tau$ was approximately 3,5 , and $10 \mu$ s, respectively. When $\tau$ is 10 $\mu$ s (about $20 \%$ of the symbol time) and the average bit error probability is $10^{-3}$, there is about a $1.2 \mathrm{~dB}$ performance degradation from the single path case (without ISI, $\tau=0 \mu \mathrm{s}$ ).

Fig. 7 shows the effect of path selection on channel sounding. In the simulation, $P_{p} / P_{d}$ was set to $8 \mathrm{~dB}, \alpha$ was set to 0.9 (the length of the average period is about ten symbols), and the threshold for path selection $\left(A_{\mathrm{th}}\right)$ was set to $10 \mathrm{~dB}$ below the peak amplitude of the coarsely estimated channel impulse response. From Fig. 7, it can be observed that channel sounding with path selection achieves almost the same performance as the theoretic BPSK case. This path selection process removes most of the noise effect in channel sounding. There is about a $3.5 \mathrm{~dB}$ gain at the average bit error probability of $10^{-3}$ between the two channel sounding methods. Therefore, path selection is absolutely necessary when the average period of the IIR filter is not long enough to reduce the noise effect in the channel sounding.

Next, we simulated the channel sounding without path selection case in a two-path slow fading channel, e.g., a channel for WLL applications [18], with the maximum Doppler frequency $f_{d}$ set to 3,5 , and $10 \mathrm{~Hz}$. The excess delay of the two-path fading channel was set to 30 chips $(\tau=3 \mu \mathrm{s})$, $P_{p} / P_{d}$ was set to $8 \mathrm{~dB}$, and $\alpha$ was set to 0.99 . The system

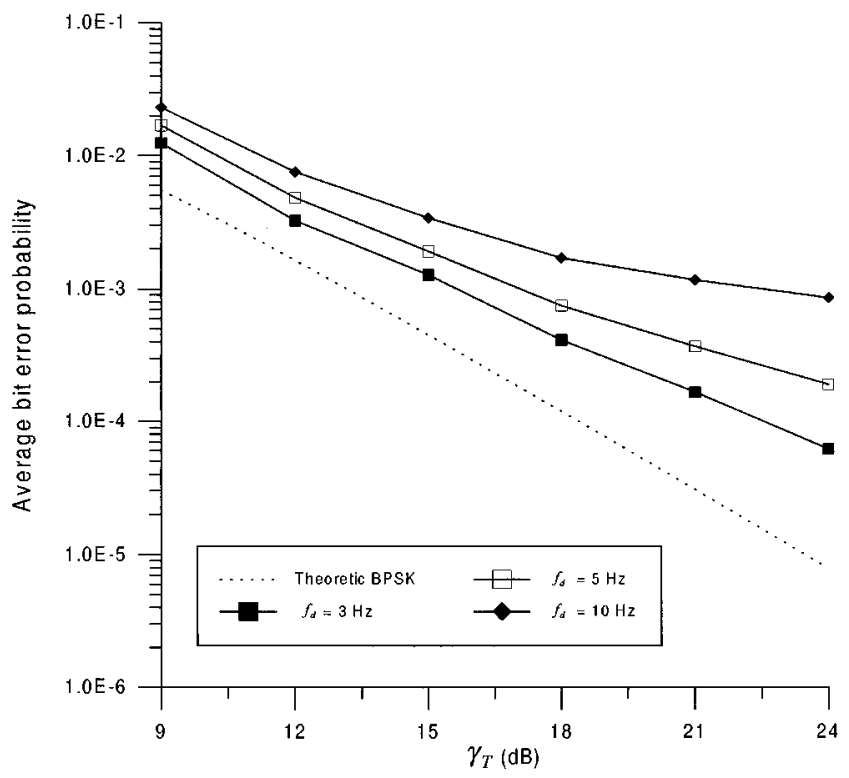

Fig. 8. Average bit error probability versus $\Upsilon_{T}$ in a two-path slow fading channel when channel sounding without path selection was used $\left(P_{p} / P_{d}=8\right.$ $\mathrm{dB}, \alpha=0.99, \tau=3 \mu \mathrm{s})$.

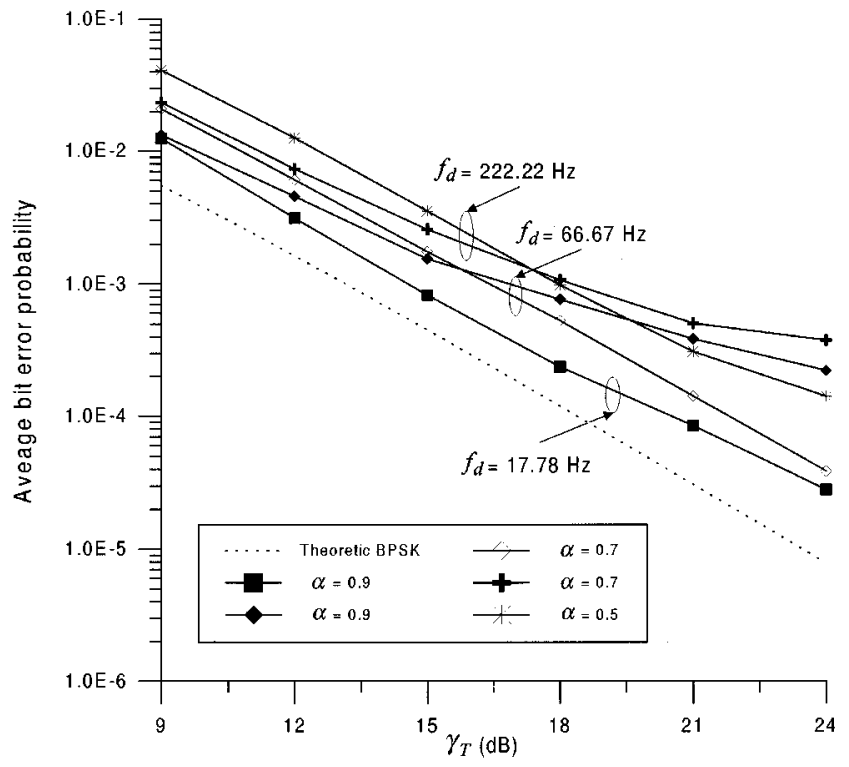

Fig. 9. Average bit error probability versus $\Upsilon_{T}$ in a two-path fast fading channel when channel sounding with path selection was used $\left(P_{p} / P_{d}=8 \mathrm{~dB}\right.$, $\tau=3 \mu \mathrm{s})$.

performance is shown in Fig. 8. As the Doppler frequency increases, the system performance degrades due to the delay in the channel estimation.

Finally, we simulated the channel sounding with the path selection case in a two-path fast fading channel; the results are shown in Fig. 9. Here, $P_{p} / P_{d}$ was again set to $8 \mathrm{~dB}$; the maximum Doppler frequency $f_{d}$ was set to $17.78,66.67$, and 222.22 $\mathrm{Hz}$; and $\tau$ was set to $3 \mu \mathrm{s}$. We observe that as Doppler frequencies increase, the time constant of the IIR filter should be decreased in order to track the channel more closely. However, a more noisy pilot signal estimation also results from a shorter time constant of the IIR filter. The noisy pilot signal estimation 
TABLE II

COMPUTATION COMPLEXITY COMPARISON

\begin{tabular}{c|c|c}
\hline Bandwidth/Window Size & $\begin{array}{c}\text { The number of } \\
\text { multiplications/symbol for } \\
\text { equivalent time domain approach }\end{array}$ & $\begin{array}{c}\text { The number of } \\
\text { multiplications/symbol for FFT } \\
\text { approach with path selection }\end{array}$ \\
\hline $10 \mathrm{MHz} / 10 \mu \mathrm{sec}$ & 55,296 & 15,872 \\
\hline $5 \mathrm{MHz} / 10 \mu \mathrm{sec}$ & 29,696 & 15,872 \\
\hline $1.25 \mathrm{MHz} / 10 \mu \mathrm{sec}$ & 10,496 & 15,872 \\
\hline $10 \mathrm{MHz} / 3 \mu \mathrm{sec}$ & 19,456 & 15,872 \\
\hline $5 \mathrm{MHz} / 3 \mu \mathrm{sec}$ & 11.776 & 15,872 \\
\hline $1.25 \mathrm{MHz} / 3 \mu \mathrm{sec}$ & 6,016 & 15,872 \\
\hline
\end{tabular}

degrades both the accuracy of channel estimation and the system performance. On the other hand, a large time constant reduces the noises in the estimated pilot signal but leads to miss tracking of channel variations. Furthermore, this miss tracking will also result in that the pilot signal cannot be effectively cancelled out at the pilot interference cancellation stage. Consequently, the uncancelled pilot signal becomes an interference itself for data signal detection, especially when the Doppler frequency is large.

\section{DISCUSSION AND CONCLUSION}

In this paper, we described an FFT-based RAKE receiver architecture for spread-spectrum downlink communications. This new RAKE receiver architecture has the following features.

1) This RAKE receiver architecture is a highly integrated solution. A sounding receiver, a spreading code matched filter, and a channel matched filter are all integrated in a single system using FFT-based matched filtering.

2) The pilot signal interference does not degrade the system performance because its effect can be removed before data signal detection.

3) The sounding receiver of this RAKE receiver architecture can respond quickly to the change of the channel. A conventional RAKE receiver usually uses a sliding correlator to search for multipath delay profiles and provide main path delays to the tracking loops. This search process induces a large estimation delay. A conventional RAKE receiver may lose track of some newly arrived or disappeared paths, and this will degrade the system performance. At contrast, this RAKE receiver induces much less estimation delay since our path searching and tracking are done on a symbol-by-symbol basis, and it can be quickly adapted to the change of the channel.

Next, we will calculate the computation complexity of the FFT-based RAKE receiver. Either FFT or IFFT needs $N \log _{2} N$ multiplications per symbol. In addition, each multiplier needs $N$ multiplications per symbol. An FFT is used to transform the received signal from the time domain to the frequency domain. The data signal process employs two multipliers (see Fig. 2). For the channel sounding without path selection (see Fig. 3), we totally used three multipliers, i.e., the total number of multiplications is $N \log _{2} N+5 N$ per symbol.
According to Fig. 4, an FFT, an IFFT, and two multipliers are used for the channel sounding with path selection. In this case, each symbol totally needs $3 N \log _{2} N+4 N$ multiplications. Based on the system parameters of our computer simulation (listed in Table I), we can calculate that the FFT-based RAKE receiver needs 7168 and 15872 multiplications per symbol for the two channel sounding methods, respectively.

An equivalent RAKE receiver can be implemented in the time domain, which employs a bank of corrlators to search and track the channel on a symbol-by-symbol basis. The search window for the multipath should cover only the region on which useful paths are likely to occur. For a wide-band CDMA system with a large cell size for mobile cellular applications, we may need a relative large window size to search/track the multipath. For example, a measurement result suggested a window size of approximately $10 \mu \mathrm{s}$ [22]. Of course, a small search window size can be used for a small cell size due to its relatively small delay spread.

In Table II, we compare the computation complexity of the two approaches at different bandwidth (chip rate) and window sizes, assuming that the processing gain is 512 . We also assumed that the number of RAKE fingers (for data detection) and the number of taps of the reconstruction filter (for pilot signal reconstruction) are both four for the equivalent time-domain approach. Table II shows that the FFT approach does not always have a lower computation complexity than the time-domain approach, but its computation advantage becomes more apparent for a wide-band system operating an environment with a large delay spread. Furthermore, this architecture is very suitable for a multicode system. As high-data-rate transmission is needed for multimedia services, one method suggested is to use multiple code channels for a single user. For a conventional receiver, each added code channel uses three to four additional fingers to detect data. With the FFT-based architecture, however, each added code channel employs only two additional multipliers for data code depreading and channel matching.

In this paper, we simulated our RAKE receiver in both a static channel and a two-path fading channel. Our simulation results show that average bit error probability of the system is close to the theoretic optimum in a static channel. In a mobile radio channel, we found that the system performance degrades as the maximum Doppler frequency increases. To optimize for 
the system performance, the time constant of the channel estimator should be adapted to the channel Doppler frequencies. Overall, our simulation results could serve as a valuable reference to other wide-band CDMA RAKE receiver designers.

For future research, multiuser detection with the FFT-based RAKE receiver in uplink transmissions can be studied. Parallel interference cancellation (PIC) techniques can be easily adopted [23] and used with the FFT-based RAKE receiver architecture. Within the PIC detector, we estimate MAI and remove the estimated MAI from the received signal in parallel in the frequency domain. We expect that through multiuser detection, both the system performance and the system capacity can be further improved.

\section{APPENDIX I}

\section{FFT-BASED CODE DESPREADING}

In this Appendix, we introduce the concept of FFT-based matched filtering and discuss how this method can be used for code despreading.

Assume that $x 1[n]$ and $x 2[n]$ are two discrete time signals of length $N(0 \leq n \leq N-1)$ and that their FFTs are $X 1[k]$ and $X 2[k]$, respectively. A sequence $x 3[n]$, whose FFT is $X 3[k]=$ $X 1[k] X 2^{*}[k]$, is the circular convolution between $x 1[n]$ and $x 2^{*}\left[((-n))_{N}\right]$ [20], [24], where the notation $((m))_{N}$ denotes ( $m$ modulo $N) \cdot x 3[n]$ can be expressed as

$$
x 3[n]=\sum_{m=0}^{N-1} x 1[m] x 2^{*}\left[((m-n))_{N}\right] .
$$

Let both $x_{1}[n]$ and $x_{2}[n]$ be $c_{d}[n]$, where $c_{d}[n]$ is the data signal spreading code. According to (A.1), the autocorrelation of $c_{d}[n]$ is

$$
\begin{aligned}
& \Gamma_{c_{d}}[n]=\mathcal{I} \mathcal{F} \mathcal{F} \mathcal{T}\left\{C_{d}[k] C_{d}^{*}[k]\right\} \\
& =\sum_{m=0}^{N-1} c_{d}[m] c_{d}^{*}\left[((m-n))_{N}\right] \\
& = \begin{cases}N & \text { when } n=0, \\
\ll N & \text { when } n \neq 0\end{cases}
\end{aligned}
$$

where $C_{d}[k]$ is the FFT of $c_{d}[n]$. Since $c_{d}[n]$ is a random code, $\Gamma_{c_{d}}[n]$ can be approximated by a delta function of height $N$. Therefore, a data code matched filter can be implemented by FFT-based matched filtering.

As FFT is a symbol-by-symbol operation, when the window of FFT computation does not synchronize with the received symbol timing, ISI appears in the FFT signal. Without loss of generality, we assume the original data signal has unit amplitude and ignore both the pilot signal and the noise term. For the $i$ th received symbol, the remaining signal after pilot interference cancellation (in time domain representation) is

$$
\begin{aligned}
\tilde{r} 1^{(i)}[n]= & d^{(i-1)} c_{d}\left[((n-\epsilon))_{N}\right](u[n]-u[n-\epsilon]) \\
& +d^{(i)} c_{d}\left[((n-\epsilon))_{N}\right](u[n-\epsilon]-u[n-N])
\end{aligned}
$$

where

$\epsilon$

time offset between the window of FFT computation and the received symbol timing; $d^{(i-1)}$ previous information bit;

$d^{(i)} \quad$ present information bit;

$u[n] \quad$ unit step function.

The cross-correlation between $\tilde{r} 1^{(i)}[n]$ and $c_{d}[n]$ is

$$
\begin{aligned}
\Gamma_{\tilde{r} 1, c_{d}}^{(i)}[n] & =\mathcal{I} \mathcal{F} \mathcal{F} \mathcal{T}\left\{\tilde{R} 1^{(i)}[k] C_{d}^{*}[k]\right\} \\
& =\sum_{m=0}^{N-1} \tilde{r} 1^{(i)}[m] c_{d}^{*}\left[((m-n))_{N}\right]
\end{aligned}
$$

where $\tilde{R} 1^{(i)}[k]$ is the FFT of $\tilde{r} 1^{(i)}[n]$. The exactly time matched point of $\Gamma_{\tilde{r} 1, c_{d}}^{(i)}[n]$ is

$$
\begin{aligned}
\Gamma_{\tilde{r} 1, c_{d}}^{(i)}[\epsilon]= & \sum_{m=0}^{N-1} \tilde{r} 1^{(i)}[m] c_{d}^{*}\left[((m-\epsilon))_{N}\right] \\
= & d^{(i-1)} \sum_{m=0}^{\epsilon-1} c_{d}\left[((m-\epsilon))_{N}\right] c_{d}^{*}\left[((m-\epsilon))_{N}\right] \\
& +d^{(i)} \sum_{m=\epsilon}^{N-1} c_{d}\left[((m-\epsilon))_{N}\right] c_{d}^{*}\left[((m-\epsilon))_{N}\right] \\
= & \begin{cases}d^{(i)} N, & \text { when } d^{(i)}=d^{(i-1)} \\
d^{(i)}(N-2 \epsilon), & \text { when } d^{(i)}=-d^{(i-1)}\end{cases}
\end{aligned}
$$

The ISI effect can be neglected if the symbol period is much larger than the time offset, i.e., $N \gg \epsilon$.

\section{APPENDIX II \\ FFT-BASED CHANNEL MATCHING}

We derive the equation for the first point of the channel matched filter output and prove that a channel matched filter is also a maximal-ratio combiner for multipath signals in this Appendix.

Without loss of generality, we assume perfect channel estimation and no noise. The relationship between the estimated channel impulse response $\tilde{h}^{(i)}[n]$ and the estimated channel frequency response $\tilde{H}^{(i)}[k]$ is expressed as

$$
\begin{aligned}
\tilde{h}^{(i)}[n] & =\mathcal{I} \mathcal{F} \mathcal{F} \mathcal{T}\left\{\tilde{H}^{(i)}[k]\right\} \\
& =\sum_{l=1}^{L} a_{l} \delta\left[n-\epsilon_{l}\right]
\end{aligned}
$$

where

$L \quad$ number of resolvable paths;

$a_{l} \quad$ complex gain of the $l$ th path;

$\epsilon_{l} \quad$ excess delay of the $l$ th path.

The data code despreading output is $\tilde{R} 1^{(i)}[k] C_{d}^{*}[k]$. Here, we ignore the pilot signal, the noise term, and the ISI effect. The IFFT of $\tilde{R} 1^{(i)}[k] C_{d}^{*}[k]$ is

$$
\begin{aligned}
\Gamma_{\tilde{r} 1, c_{d}}^{(i)}[n] & =\mathcal{I} \mathcal{F} \mathcal{F} \mathcal{T}\left\{\tilde{R} 1^{(i)}[k] C_{d}^{*}[k]\right\} \\
& =\mathcal{I} \mathcal{F} \mathcal{F} \mathcal{T}\left\{d^{(i)} H^{(i)}[k]\left|C_{d}[k]\right|^{2}\right\} \\
& =d^{(i)} \sum_{l=1}^{L} a_{l} \Gamma_{c_{d}}\left[\left(\left(n-\epsilon_{l}\right)\right)_{N}\right] .
\end{aligned}
$$

We obtain a frequency-domain channel matching output by multiplying $\tilde{R} 1^{(i)}[k] C_{d}^{*}[k] \quad\left(\tilde{R} 1^{(i)}[k] C_{d}^{*}[k]=\right.$ 
$d^{(i)} H^{(i)}[k]\left|C_{d}[k]\right|^{2}$ ) with the complex conjugate of the estimated channel frequency response $\tilde{H}^{*(i)}[k]$. The time-domain channel matching output $\Gamma_{\mathrm{ch}}^{(i)}[n]$ is the IFFT of the frequency-domain channel matching output $\tilde{R} 1^{(i)}[k] C_{d}^{*}[k] \tilde{H}^{*(i)}[k]$ and can be expressed by the circular convolution of $\Gamma_{\tilde{r} 1, c_{d}}^{(i)}[n]$ and $\tilde{h}^{*(i)}\left[((-n))_{N}\right]$, i.e.,

$$
\begin{aligned}
\Gamma_{\mathrm{ch}}^{(i)}[n] & =\mathcal{I} \mathcal{F} \mathcal{F} \mathcal{T}\left\{\tilde{R} 1^{(i)}[k] C_{d}^{*}[k] \tilde{H}^{*(i)}[k]\right\} \\
& =\mathcal{I} \mathcal{F} \mathcal{F} \mathcal{T}\left\{d^{(i)} H^{(i)}[k]\left|C_{d}[k]\right|^{2} \tilde{H}^{*(i)}[k]\right\} \\
& =\sum_{m=0}^{N-1} \Gamma_{\tilde{r} 1, c_{d}}^{(i)}[m] \tilde{h}^{*(i)}\left[((m-n))_{N}\right] .
\end{aligned}
$$

By setting $n=0$ into (B.3), the first point of $\Gamma_{\mathrm{ch}}^{(i)}[n]$ is

$$
\Gamma_{\mathrm{ch}}^{(i)}[0]=\sum_{m=0}^{N-1} \Gamma_{\tilde{r} 1, c_{d}}^{(i)}[m] \tilde{h}^{*(i)}[m] .
$$

Substituting (B.1) and (B.2) into (B.4) yields

$$
\begin{aligned}
\Gamma_{\mathrm{ch}}^{(i)}[0] & =d^{(i)} \sum_{m=0}^{N-1} \sum_{n=1}^{L} \sum_{l=1}^{L} a_{n} \Gamma_{c_{d}}\left[\left(\left(m-\epsilon_{n}\right)\right)_{N}\right] a_{l}^{*} \delta\left[m-\epsilon_{l}\right] \\
& =d^{(i)} \sum_{n=1}^{L} \sum_{l=1}^{L} a_{n} a_{l}^{*} \Gamma_{c_{d}}\left[\left(\left(\epsilon_{l}-\epsilon_{n}\right)\right)_{N}\right] \\
& \approx d^{(i)} N \sum_{l=1}^{L}\left|a_{l}\right|^{2} .
\end{aligned}
$$

Therefore, the channel matched filter is a maximal-ratio combiner for multipath signals, and only the first point of $\Gamma_{\mathrm{ch}}^{(i)}[n]$ is needed for data decision.

\section{REFERENCES}

[1] A. Baier, U.-C. Fiebig, W. Granzow, W. Koch, P. Teder, and J. Thielecke, "Design study for a CDMA-based third-generation mobile radio system," IEEE J. Select. Areas Commun., vol. 12, pp. 733-743, May 1994.

[2] F. Ovesijö, E. Dahlman, T. Ojanperä, A. Toskala, and A. Klein, "FRAMES multiple access mode 2-Wideband CDMA," in Proc. PIMRC'97, Helsinki, Sept. 1997, pp. 42-46.

[3] F. Adachi, M. Sawahashi, and H. Suda, "Wideband DS-CDMA for nextgeneration mobile communication systems," IEEE Commun. Mag., vol 36, pp. 56-69, Sept. 1998.

[4] W. C. Y. Lee, "Overview of cellular CDMA," IEEE Trans. Veh. Technol., vol. 40, pp. 291-302, May 1991.

[5] K. S. Gilhousen, I. M. Jacobs, R. Padovani, A. J. Viterbi, A. Weaver Jr., and C. E. Wheatley, "On the capacity of a cellular CDMA system," IEEE Trans. Veh. Technol., vol. 40, pp. 303-312, May 1991.

[6] G. L. Turin, "Introduction to spread-spectrum antimultipath techniques and their application to urban digital radio," Proc. IEEE, vol. 68, pp 328-353, Mar. 1980.

[7] H. Ochsner, "Direct-sequence spread-spectrum receiver for communication on frequency-selective fading channels," IEEE J. Select. Areas Commun., vol. SAC-5, pp. 188-193, Feb. 1987.

[8] G. J. R. Povey, P. M. Grant, and R. D. Pringle, "A decision-directed spread-spectrum RAKE receiver for fast-fading mobile channels," IEEE Trans. Veh. Technol., vol. 45, pp. 491-502, Aug. 1996.

[9] H. Andoh, M. Sawahashi, and F. Adachi, "Channel estimation using time multiplex pilot symbols for coherent RAKE combining for DS-CDMA mobile radio," in Proc. PIMRC'97, Helsinki, Finland, Sept. 1997, pp. 954-958.
[10] E. D. Re, R. Fantacci, and P. Giannoccaro, "Practical RAKE receiver architecture for downlink communications in a DS-CDMA mobile system," Proc. Inst. Elect. Eng. Commun., vol. 145, pp. 277-282, Aug. 1998.

[11] C. D'Amours, M. Moher, and A. Yongacoglu, "Comparison of pilot symbol-assisted and differentially detected BPSK for DS-CDMA systems employing RAKE receivers in Rayleigh fading channels," IEEE Trans. Veh. Technol., vol. 47, pp. 1258-1267, Nov. 1998.

[12] H. Huang, C.-L. I, and S. T. Brink, "Improving detection and estimation in pilot-aided frequency selective CDMA channels," in Proc. ICUPC'97, San Diego, CA, Oct. 1997, pp. 198-201.

[13] M. B. Pursley, "Performance evaluation of phase-coded spread-spectrum multiple-access communication-Part I," IEEE Trans. Commun. vol. COM-25, pp. 795-799, Aug. 1977.

[14] W. C. Jakes Jr., Microwave Mobile Communications. New York: Wiley-Interscience, 1974

[15] A. P. Hulbert, "Comprehensive RAKE-A novel and practical receiver architecture offering improved performance," in Proc. ISSSTA'94, Oulu, Finland, July 1994, pp. 470-474.

[16] T. S. Rappaport, Wireless Communications, Principles \& Practice. Englewood Cliffs, NJ: Prentice-Hall, 1996.

[17] W. Mohr, "Radio propagation for local loop applications at $2 \mathrm{GHz}$," in Proc. UPC'94, San Diego, CA, Oct. 1994, pp. 119-123.

[18] T. Westman, K. Rikkinen, and T. Ojanperä, "DCS 1800 based wireless local loop (WLL)," in Proc. VTC'96, Atlanta, GA, Apr. 1996, pp. 313-315.

[19] J. Kilvington and F. England, "Receivers for navigation satellite systems," 4601005.

[20] E. O. Brigham, The Fast Fourier Transform and Its Applications Englewood Cliffs, NJ, 1988

[21] S. Glisic and B. Vucetic, Spread Spectrum CDMA Systems for Wireless Communications. Boston, MA: Artech House, 1997.

[22] K. A. Stewart, G. P. Labedz, and K. Sohrabi, "Wideband channel mearments at $900 \mathrm{~Hz}$," in Proc. VTC'95, Chicago, July 1995, pp. 236-240.

[23] S. Moshavi, "Multi-user detection for DS-CDMA communications," IEEE Commun. Mag., vol. 34, pp. 124-136, Oct. 1996.

[24] A. V. Oppenheim and R. W. Schaferm, Discrete-Time Signal Processing. Englewood Cliffs, NJ: Prentice-Hall, 1989.

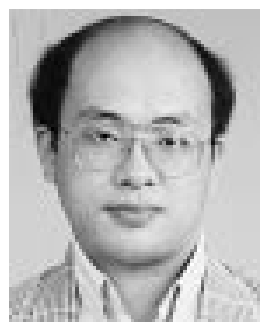

Shin-Yuan Wang was born in Hsin-Chu, Taiwan, R.O.C. He received the B.S. degree is electrical engineering from National Taiwan Ocean University in 1993 and the M.S. degree in communication engineering from the National Chiao-Tung University, Taiwan, in 1995, where he is currently pursuing the Ph.D. degree.

His research interests include the design of baseband receiver architectures for spread-spectrum systems and smart antenna systems.

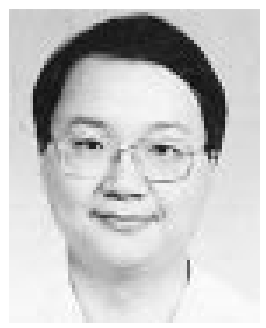

Chia-Chi Huang was born in Taiwan, R.O.C. He received the B.S. degree in electrical engineering from National Taiwan University in 1977 and the M.S. and $\mathrm{Ph} . \mathrm{D}$. degrees in electrical engineering from the University of California, Berkeley, in 1980 and 1984, respectively.

From 1984 to 1988, he was an FR and Communication System Engineer with the Corporate Research and Development Center, General Electric Co., Schenectady, NY, where he worked on mobile radio communications. From 1989 to 1992 , he was with the IBM T. J. Watson Research Center, Yorktown Heights, NY, as a Research Staff Member, working on indoor radio communications. Since 1992, he has been with the Department of Communication Engineering, National Chiao Tung Unversity, Hsinchu, Taiwan, as an Associate Professor. 